\title{
A 41-gene signature derived from breast cancer stem cells as a predictor of survival
}

\author{
Zhi-Qiang Yin ${ }^{1+}$, Jian-Jun Liư ${ }^{1 \dagger}$, Ying-Chun Xu', Jian Yu², Guo-Hui Ding ${ }^{2}$, Feng Yang ${ }^{1}$, Lei Tang ${ }^{1}$, Bao-Hong Liu², \\ Yue Ma', Yu-Wei Xia', Xiao-Lin Lin' ${ }^{1}$ and Hong-Xia Wang ${ }^{1,3^{*}}$
}

\begin{abstract}
Purpose: The aim of this study was to evaluate the ability of a 41-gene signature derived from breast cancer stem cells (BCSCs) to estimate the risk of metastasis and survival in breast cancer patients.

Methods: The centroid expression of the 41-gene signature derived from BCSCs was applied as the threshold to classify patients into two separate groups - patients with high expression (high-EL) of the prognostic signature and patients with low expression (low-EL). The predictive ability of the 41-gene signature was evaluated by Cox regression model and was compared against other popular tests, such as Oncotype and MammaPrint.

Results: Our results showed that the 41-gene prognostic signature was significantly associated with age $(P=.0351)$ and ER status $(P=.0095)$. The analysis indicated that patients in the high-EL group had a worse prognosis than those in the low-EL group in terms of both overall survival (OS: HR, 2.05, $P=.009)$ and distant metastasis-free survival (DMFS: HR, 2.24, $P=.002$ ). Additionally, the 41-gene signature was an independent risk factor and separates patients based on estrogen receptor status. While comparable to Oncotype, the analysis demonstrated that the 41-gene signature had a better prognostic value in predicting DMFS and OS than AOL, NPI, St. Gallen, Veridex, and MammaPrint.

Conclusions: This study confirms the utility of the 41-gene signature and adds to the growing evidence that gene expression signatures of BCSCs have clinical potential to predict patient outcome and aid in treatment choice.
\end{abstract}

Keywords: Prognostic signature, Breast cancer, Stem cell

\section{Introduction}

Personalized medicine, the selection of therapy based on a patient's individual characteristics, may result in better outcomes than the use of generalized medicine [1-4]. Prognostic factors commonly applied in breast cancer include age, tumor size, lymph node involvement, pathological grade, and status of HER-2, Ki-67, and several hormone receptors, including both estrogen receptor (ER) and progesterone receptor (PR) $[5,6]$. Although several guidelines have been developed to assist clinicians in selecting patients who are at high risk of recurrence, it still remains a challenge to distinguish patients who

\footnotetext{
* Correspondence: whx365@126.com

${ }^{\dagger}$ Equal contributors

'Shanghai Renji Hospital, Shanghai Jiaotong University School of Medicine, Shanghai 200127, China

${ }^{3}$ Department of Oncology, Renji Hospital, School of Medicine, Shanghai Jiaotong University, Shanghai 200127, China

Full list of author information is available at the end of the article
}

have poor prognosis and require demanding adjuvant systemic therapy from those who could be spared such treatment. Due to the complexity of the disease, several other factors have been investigated for their potential to predict breast cancer outcome. However, most have only limited predictive power $[7,8]$.

Recent findings support the concept that a rare population of cells, termed cancer stem-like cells (CSCs), is the cellular origin of cancer $[9,10]$. Such findings imply that it is these CSCs that are responsible for tumor initiation, progression, and response to therapy [11,12]. Therefore, an advance in our knowledge of the properties of CSCs has become a topic of considerable interest.

We previously identified a rare population of breast cancer stem cells (BCSCs) from tissue [13,14]. Human cancer is characterized by high heterogeneity in gene expression and phenotype, both of which influence tumor growth rate and drug sensitivity. We performed expression 
profiling to identify signaling pathways enriched in BCSCs. According to the gene expression profile, we found that sixty-three probe sets corresponding to forty-one genes showed greater than a four-fold difference in BCSCs compared to non-BCSCs. We hypothesized that this BCSC signature might be useful as a classification system since it outperformed most other clinical variables in predicting the likelihood of distant metastases and overall survival (OS) in breast cancer patients.

A more accurate means of prognostication in breast cancer will improve the selection of patients for adjuvant systemic therapy and will improve clinical decisions and strategies used to treat patients with this disease. Therefore, the present study was conducted to further evaluate the forty-one gene signature as a tool to accurately estimate the risks of metastases and survival in breast cancer patients.

\section{Methods}

\section{Database of patients}

Normalized gene expression data, together with the patient's characteristics, were retrieved from the public GEO database (http://www.ncbi.nlm.nih.gov/geo; accession number GSE7390). For each patient, the information generated from the dataset included surgery type, angioinvasion (lymph vascular invasion), histopathological grading, ER status, OS, distant metastasis-free survival (DMFS), clinical risk group according to St. Gallen criteria, National Provider Identifier (NPI) criteria, Adjuvant online (AOL) (http:// www.adjuvantonline.com), Veridex signature, MammaPrint, and Oncotype Dx.

\section{Study design}

The 41 DEGs (differential expressed genes) correspond to 63 probe sets. Based on these probe sets, we obtained relevant expression values of patients from GSE7390. The centroid expression of these probe sets was applied as the patient classification threshold. Based on the threshold of the prognostic signatures, breast cancer patients in the dataset can be classified into two separate groups-patients with high expression (high-EL) of the prognostic signature and patients with low expression (low-EL) of the prognostic signature.

\section{Statistical analysis}

To assess the prognostic value of the 41-gene signature, we utilized the Kaplan-Meier estimator to plot survival curves and the log-rank test to compare differences between two groups [15]. Fisher's exact test was employed to investigate the relevance between the 41-gene signature and clinical factors. Standard Cox proportional hazards regression were implemented to predict OS and DMFS. The performance of the 41-gene signature and other standard criteria, including AOL, NPI, St. Gallen,
Veridex, Oncotype DX, and MammaPrint were evaluated in terms of LHR and Akaike information criterion (AIC) in a full model (all systems included) and in a series of reduced models where each interested factor was removed once each time. When removed from the full model, the best option results in the largest drop in LHR $\chi^{2}$ and an increase in AIC. All statistical analyses were performed by the $\mathrm{R}$ programming package with rms.

End points considered in this study were time from diagnosis to distant metastases (DMFS) and OS, which was defined as time from diagnosis to death by any cause. The linearity of the relation between the relative hazard ratio and the diameter of the tumor, age, and ER expression level were tested using the Wald test for nonlinear components of restricted cubic splines. No evidence for nonlinearity was found $(P=.83$ for age, $P=.75$ for tumor diameter, $P=.65$ for the number of positive nodes, and $P=.27$ for ER expression). We evaluated whether the hazard ratio was proportional using the method of Grambsch and Therneau.

\section{Results}

\section{Characteristics of patients}

The study was carried out with frozen archived tumor material from early stage breast cancer patients using the Affymetrix HG-U133A chip as previously described by the TRANSBIG consortium [16].

\section{Pattern of the 41-gene expression profile in breast cancer patients}

Functional annotation of these 41 genes (Table 1) provides insight into the underlying biological mechanism leading to breast cancer tumorigenesis and the cellular signaling pathways regulating BCSCs.

The gene-expression values of the 41 markers for all 198 tumors in this study are shown in Figure 1. As shown in Figure 1A, red indicates increased mRNA expression in the tumor compared to the reference; green indicates low level expression. The dotted line represents the previously determined threshold between a goodprognosis signature and a poor-prognosis signature. Tumors are rank-ordered according to the expression level of the 41 prognostic genes in tumors from 198 patients. Figure $1 \mathrm{~B}$ shows the time in years to distant metastasis as a first event of this occurrence, as well as the total duration of follow-up for all patients. Figure $1 \mathrm{C}$ shows the living status of these breast cancer patients.

\section{Association between the 41-gene prognostic signature and clinical variables}

The 198 patients were divided into two groups based on high expression level (high-EL, $\mathrm{n}=99$ ) and low expression level (low-EL, $n=99$ ), similar to earlier reports [17]. These levels correspond to a poor prognostic signature 
Table 1 List and functional annotation of the $\mathbf{4 1}$ genes in the study

\begin{tabular}{|c|c|c|}
\hline ID & Gene name & Function \\
\hline 23586 & DDX58 & DEAD box protein. \\
\hline 1041 & CDSN & Corneocdesmosin, is a secreted protein found in corneodesmosomes. \\
\hline 259230 & SGMS1 & Sphingomyelin synthase 1. \\
\hline 81669 & LOC643556 & Similar to Aurora kinase A-interacting protein (AURKA-interacting protein). \\
\hline 54809 & SAMD9 & A sterile alpha motif domain-containing protein, regulating cell proliferation/apoptosis. \\
\hline 6352 & CCL5 & Chemokine ( $\mathrm{C}-\mathrm{C}$ motif) ligand 5. \\
\hline 90362 & FAM110B & Family with sequence similarity 110 , member $B$. \\
\hline 4176 & MCM7 & DNA replication licensing factor, Minichromosome maintenance complex component 7. \\
\hline 4938 & OAS1 & $\begin{array}{l}\text { Encodes a member of the } 2-5 \mathrm{~A} \text { synthetase family, essential proteins involved in the innate } \\
\text { immune response to viral infection. }\end{array}$ \\
\hline 4939 & OAS2 & A member of the $2-5 \mathrm{~A}$ synthetase family. \\
\hline 27289 & RND1 & A small ( $\sim 21 \mathrm{kDa}$ ) signaling $\mathrm{G}$ protein, and is a member of the Rho family of GTPases. \\
\hline 3909 & LAMA3 & Laminin, alpha 3. \\
\hline 10268 & RAMP3 & Receptor (G protein-coupled) activity modifying protein 3. \\
\hline 5514 & PPP1R10 & A protein with similarity to a rat protein that has an inhibitory effect on protein phosphatase- 1 . \\
\hline 6324 & SCN1B & Sodium channel, voltage-gated, type I, beta. \\
\hline 9687 & GREB1 & An estrogen-responsive gene. \\
\hline 11151 & CHRO1A & Coronin, actin binding protein, $1 \mathrm{~A}$. \\
\hline 3434 & IFIT1 & Interferon-induced protein with tetratricopeptide repeats 1. \\
\hline 3433 & IFIT2 & Interferon-induced protein with tetratricopeptide repeats 2. \\
\hline 3437 & IFIT3 & Interferon-induced protein with tetratricopeptide repeats 3. \\
\hline 634 & CEACAM 1 & Carcinoembryonic antigen-related cell adhesion molecule 1 (biliary glycoprotein). \\
\hline 4680 & CEACAM6 & Carcinoembryonic antigen-related cell adhesion molecule 6. \\
\hline 79971 & WLS & wntless homolog (Drosophila). \\
\hline 3456 & IFNB1 & Interferon, beta 1, fibroblast. \\
\hline 9442 & MED27 & Mediator complex subunit 27, the activation of gene transcription. \\
\hline 8638 & OASL & 2'-5'-oligoadenylate synthetase-like gene. \\
\hline 1316 & KLF6 & A member of the Kruppel-like family of transcription factors. \\
\hline 55422 & ZNF331 & A zinc finger protein containing a KRAB (Kruppel-associated box) domain. \\
\hline 3853 & KRT6A & A member of the keratin gene family. \\
\hline 653 & BMP5 & A member of the bone morphogenetic protein family. \\
\hline 10916 & MAGED2 & Melanoma-associated antigen D2. \\
\hline 3627 & CXCL10 & A chemokine of the CXC subfamily and ligand for the receptor CXCR3. \\
\hline 3433 & $\mathrm{H} \| \mathrm{H} 2$ & Interferon induced with helicase C domain 2. \\
\hline 3569 & IL6 & Interleukin 6. \\
\hline 3576 & IL8 & Interleukin 8. \\
\hline 347733 & TUBB2B & A beta isoform of tubulin, which binds GTP and is a major component of microtubules. \\
\hline 629 & CFB & Complement factor B. \\
\hline 56999 & ADAMTS9 & A disintegrin and metalloproteinase with thrombospondin motifs protein family. \\
\hline 6482 & HS.374257 & ST3 beta-galactoside alpha-2,3-sialyltransferase 1. \\
\hline 90627 & STARD13 & StAR-related lipid transfer (START) domain containing 13. \\
\hline 64135 & $\mathrm{IFIH} 1$ & Interferon induced with helicase C domain 1. \\
\hline
\end{tabular}




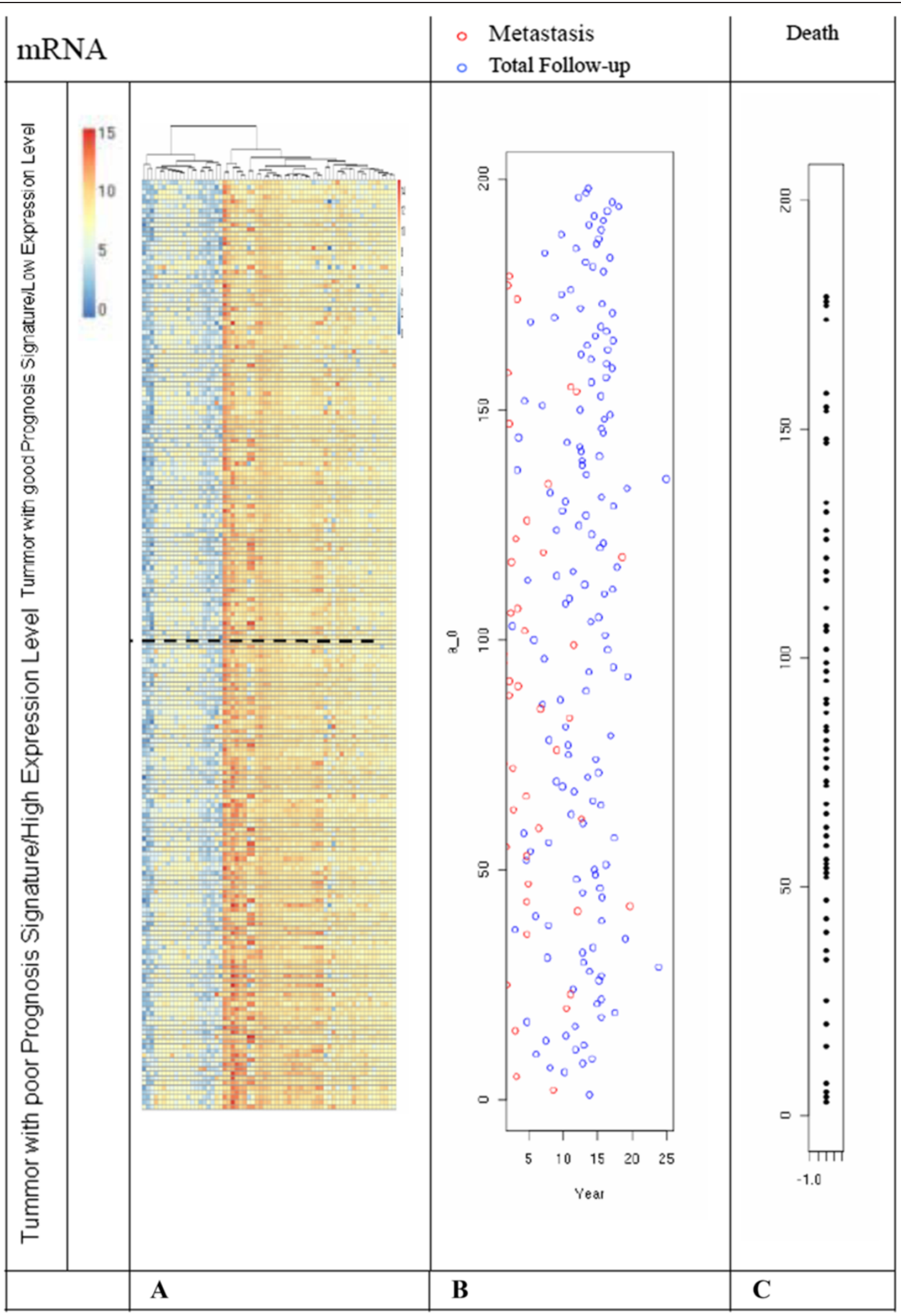

Figure 1 (See legend on next page.) 
(See figure on previous page.)

Figure 1 Pattern of expression of genes used to determine the prognosis and clinical characteristics of 198 breast cancer patients. Panel A shows the pattern of expression of the 63 marker genes in a series of 198 consecutive patients with breast carcinoma. Each row represents the prognostic profile of the 63 marker genes for one tumor, and each column represents the relative level of expression of one gene. The tumors are numbered from 1 to 198 on the $y$ axis, and the genes are numbered from 1 to 63 on the $x$ axis. Panel $\mathbf{B}$ shows the time in years to distant metastasis as a first event for those in whom this occurred, and the total duration of follow-up for all other patients. Panel $\mathbf{C}$ shows the living status. The black dots represent the number of patients who died.

and a good prognostic signature, respectively. To gain insight into the relationship between the 41-gene prognostic signature and clinical variables, we performed correlation analysis with histopathologic data of patients, such as, age, surgery type, grade, and ER expression as determined by immunohistochemical (IHC) staining. The results showed that the 41-gene prognostic signature was significantly associated with age $(P=.0351)$ and ER status $(P=.0095)$. Patients in the high-EL group were younger in age and had ER-negative tumors. There was also a slightly significant association with tumor grade. However, the $\mathrm{p}$ value showed no statistical significance.

\section{Analysis of DMFS and OS based on the prognostic signature} Our analysis indicated that the likelihood of patients developing distant metastasis at 5 years and 10 years was higher in the low-EL group than in the high-EL group
(5 year DMFS: $88 \%$ versus $75 \%$, respectively; 10 years DMFS: $83 \%$ versus $64 \%$, respectively). Prolonged OS was also observed in low-EL patients.

Additionally, multivariate analysis was conducted to adjust for confounding variables including age, tumor size, tumor grade, and ER status. Results confirmed that the 41-gene signature was an independent prognostic factor for these breast cancer patients (OS: HR, 1.96, $P=.02$; DMFS: HR, 2.09, $P=.008)$.

\section{Survival comparison between the new markers and other standard criteria}

The Kaplan-Meier curve (Figure 2A) showed a significant difference (HR, 2.236; 95\% confidence interval [CI], 1.319 to 3.79 ) in the probability that patients would remain metastasis-free in the low-EL compared to the high-EL group $(P=.002)$. The 41-gene prognostic
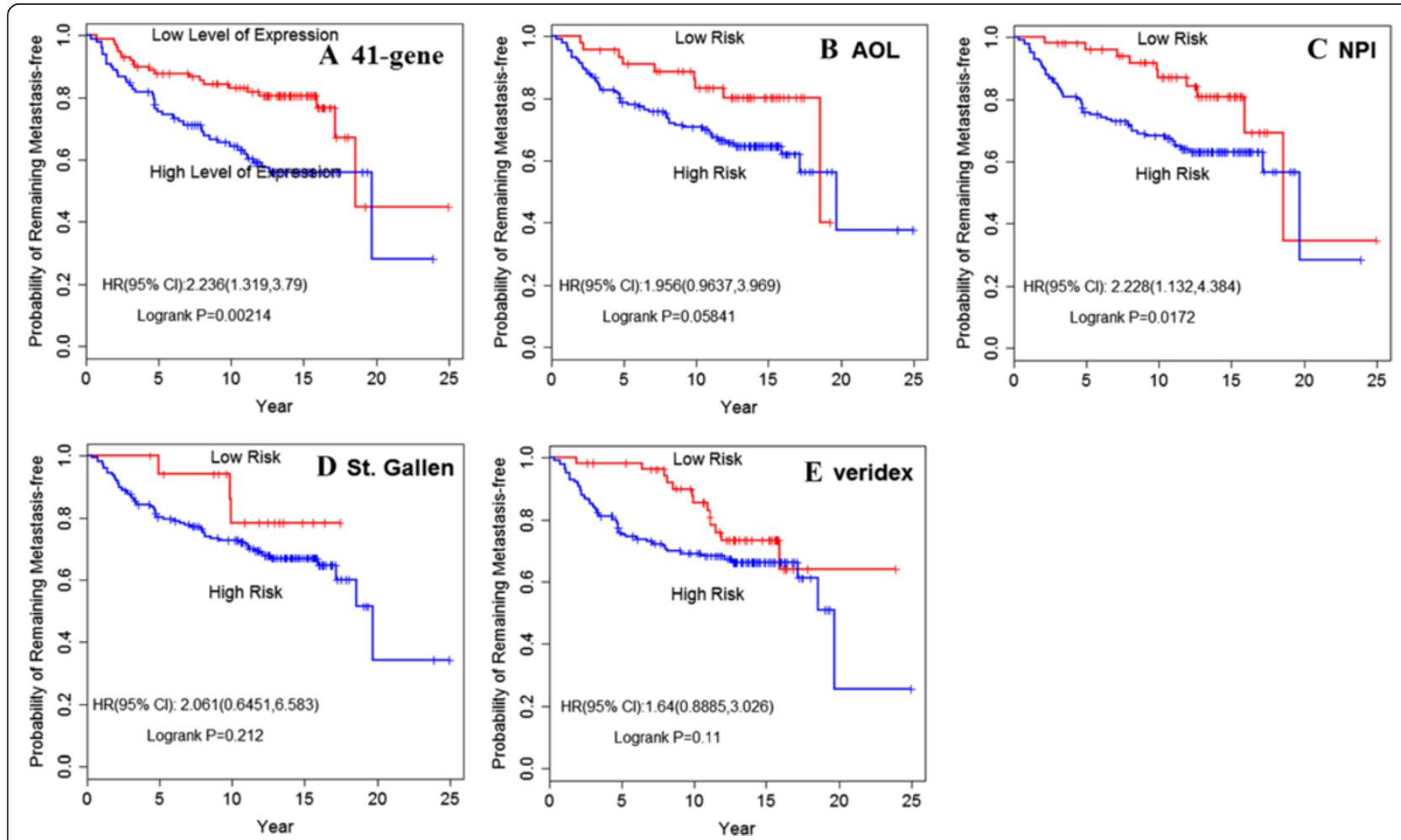

Figure 2 Kaplan-Meier analysis of the probability that patients would remain free of distant metastasis among all patients. A. prediction value of DMFS by the 41-gene signature. Patients were divided into those with a good-prognostic signature and those with a poor prognostic signature according to gene-expression profiling; $\mathbf{B}$. prediction value of OS by AOL consensus criteria; $\mathbf{C}$. prediction value of DMFS by NPI consensus criteria; D. prediction value of DMFS by St. Gallen criteria; E. prediction of Veridex signature. The $p$ values were calculated by log-rank test. 
signature was also extremely useful in predicting the outcome of OS (HR, 2.050; 95\% CI, 1.186 to $3.545 ; P=$ 0.009) (Figure 3A).

To obtain a more powerful estimate of the signature in predicting clinical outcome, we compared the 41-gene prognostic signature with other commonly used criteria, such as AOL, NPI, St. Gallen, and Veridex. Based on this analysis, patients in the database can be divided into a high-risk group and a low-risk group according to various histologic and clinical characteristics. We calculated DMFS and OS according to these different prognostic profiles. The analysis indicated that the 41-gene signature had the best prognostic value in predicting DMFS $(P=.058$ for AOL; $P=0.017$ for NPI; $P=.11$ for Veridex; and $P=.212$ for St. Gallen) (Figure 2B, 2C, 2D, 2E) and OS $(P=.074$ for AOL; $P=.031$ for NPI; $P=.053$ for Veridex; and $P=.312$ for St. Gallen) for early breast cancer patients (Figure 3B, 3C, 3D, 3E).

\section{Prognostic value in high-risk patients defined by other standard criteria}

The 41-gene prognostic signature was also highly predictive of the risk of DMFS and OS among the subgroup of patients, which were thought to be high risk according to other existing criteria. As shown in the KaplanMeier curves, we found significant differences in the probability of remaining metastasis-free between the high-EL signature and the low-EL signature, even though all were assigned to the high-risk group based on other criteria $(P=.001$ for AOL; $P=.001$ for NPI; $P=.049$ for Veridex; $P=.004$ for St. Gallen; $P=.006$ for
MammaPrint; and $P=.018$ for Oncotype Dx) (Figure 4A, 4B, 4C, 4D; Figure 5C, 5F). A similar trend was observed when assessing OS (Figure 4E, 4F, 4G, 4H; Figure 5B, 5E). Thus, the new prognostic signature more accurately predicts breast cancer survival rate (or metastasis) than other histologic and clinical characteristics. These results highlight the value of the prognosis profile and the robustness of the profiling technique.

\section{Comparison of the prognostic value of the 41-gene signature with Oncotype Dx and MammaPrint}

To assess the concordance of the 41-gene signature with published prognostic gene signatures, we implemented the original algorithms of the Oncotype Dx (Genomic Health) and MammaPrint (Agendia) gene signatures and applied them to the 41-gene signature in our compendium of microarray datasets.

Using data from the 198 patients with node-negative tumors, we analyzed the prognostic value of the 41-gene signature, Oncotype Dx, MammaPrint, and other criteria (Table 2). The results of multivariate analysis indicated that there was significant prognostic power for the 41gene signature $(P=.03)$ and Oncotype Dx $(P=.002)$. However, there was no statistically significant difference observed for the analysis using MammaPrint $(P=.647)$, AOL criteria $(P=.551)$, NPI criteria $(P=.16)$, St. Gallen criteria $(P=.383)$, or Veridex criteria $(P=.335)$.

We further investigated the prognostic ability of the 41-gene signature under different definitions of "high risk" using forest plots. As shown in Figure 5A and Figure 5D,
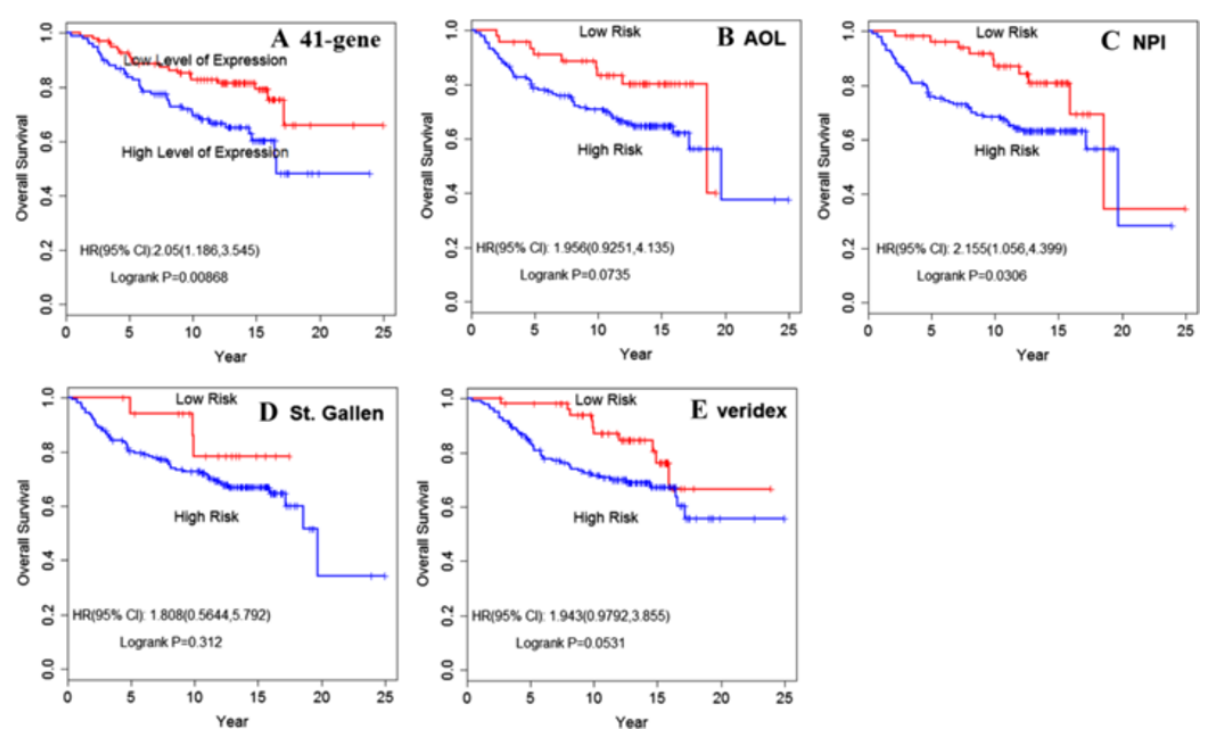

Figure 3 Kaplan-Meier analysis of the probability of OS. A. prediction value of OS by the 41-gene signature. Patients were divided into those with a good-prognostic signature and those with a poor prognostic signature according to gene-expression profiling; $\mathbf{B}$. prediction value of OS by AOL consensus criteria; $\mathbf{C}$. prediction value of OS by NPI consensus criteria; $\mathbf{D}$. prediction value of OS by St. Gallen criteria; $\mathbf{E}$. Prediction value of Veridex signature. The $p$ values were calculated by log-rank test. 

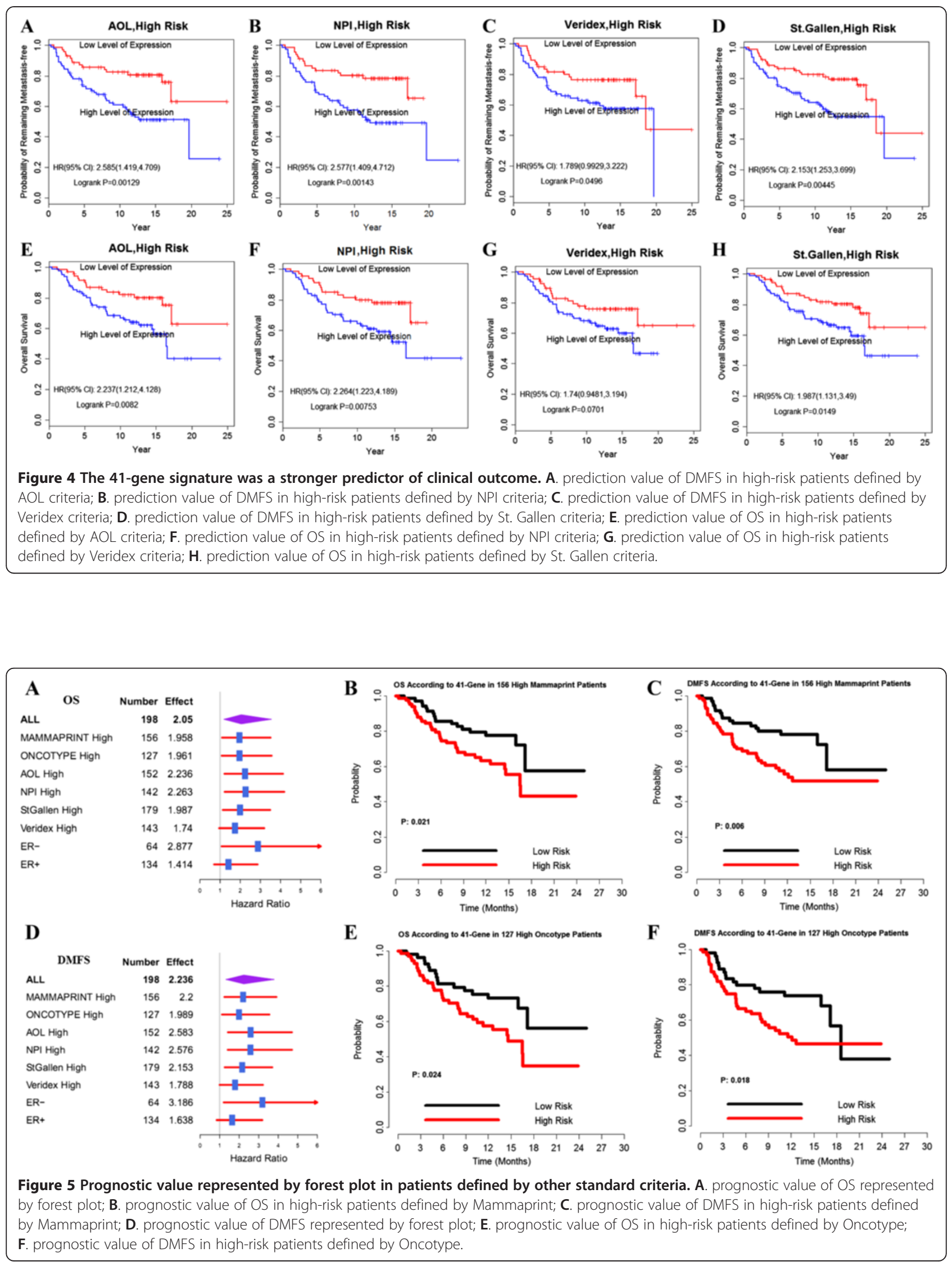
Table 2 Comparison of the prognostic value of 41-gene signature with other risk assessment criteria

\begin{tabular}{|c|c|c|c|c|c|c|}
\hline & \multicolumn{3}{|c|}{ OS } & \multicolumn{3}{|c|}{ DMFS } \\
\hline & $\Delta$ LHR & P-value & $\triangle \mathrm{AIC}$ & $\Delta$ LHR & P-value & $\triangle \mathrm{AIC}$ \\
\hline Signature & -4.929 & $0.03^{*}$ & 2.929 & -10.513 & $0.008^{*}$ & 5.475 \\
\hline ONCOTYPE & -13.286 & $0.002^{*}$ & 11.286 & -13.734 & $0.004^{*}$ & 8.696 \\
\hline MAMMAPRINT & -0.221 & 0.647 & -1.779 & -3.038 & 0.986 & -2 \\
\hline $\mathrm{AOL}$ & -0.377 & 0.551 & -1.623 & -3.325 & 0.601 & -1.713 \\
\hline NPI & -3.658 & 0.16 & 2.764 & -6.823 & 0.131 & 2.756 \\
\hline St. Gallen & -0.724 & 0.383 & -1.276 & -3.33 & 0.582 & -1.708 \\
\hline Veridex & -0.991 & 0.335 & -1.009 & -3.987 & 0.343 & -1.051 \\
\hline
\end{tabular}

The independent contribution of each interested factor to patient outcome was assessed by first removing the factors concerned and then calculating the difference of LHR and AIC. A larger drop of LHR and an increase in AIC indicate a higher significance of the removed system.

$\Delta \mathrm{LHR}$, change of likelihood ratio between full model fitting and one concerned system removed; $\triangle \mathrm{AIC}$, change of Akaike information criterion between full model fitting and one concerned system removed; *, statistical significant.

the new markers displayed good predictive ability in almost all subgroups except for ER-positive patients.

\section{Subgroup analysis according to ER status}

In order to discuss the impact of ER status on the 41gene signature, we separately analyzed the predictive value of these markers in ER-positive and ER-negative patients. The survival curves were statistically significantly different between the high-EL patients and lowEL patients for DMFS $(P=.014)$ and OS $(P=.028)$ in ER negative patients, indicating a good predictive ability in this subgroup (Figure 6A, 6B). However, the signature did not show strong predictive ability for ER positive patients (Figure 6C, 6D). These curves confirmed earlier results from forest plot analysis (Figure 5A, 5D).

\section{Discussion}

Previous studies linking gene expression profiles to clinical outcome in breast cancer have demonstrated that the potential for distant metastasis and OS probability may be attributable to biological characteristics of the primary tumor [18-21]. In their seminal work, Paik et al. [22] reported that a 21-gene recurrence score (RS) assay quantifies the likelihood of distant recurrence in women with ER-positive, lymph node-negative breast cancer treated with adjuvant tamoxifen; it also predicts the magnitude of chemotherapy benefit. Perou et al. [23] identified tumors with distinct patterns of gene expression termed "basal type" and "luminal type", using complementary DNA (cDNA) microarray to analyze breast
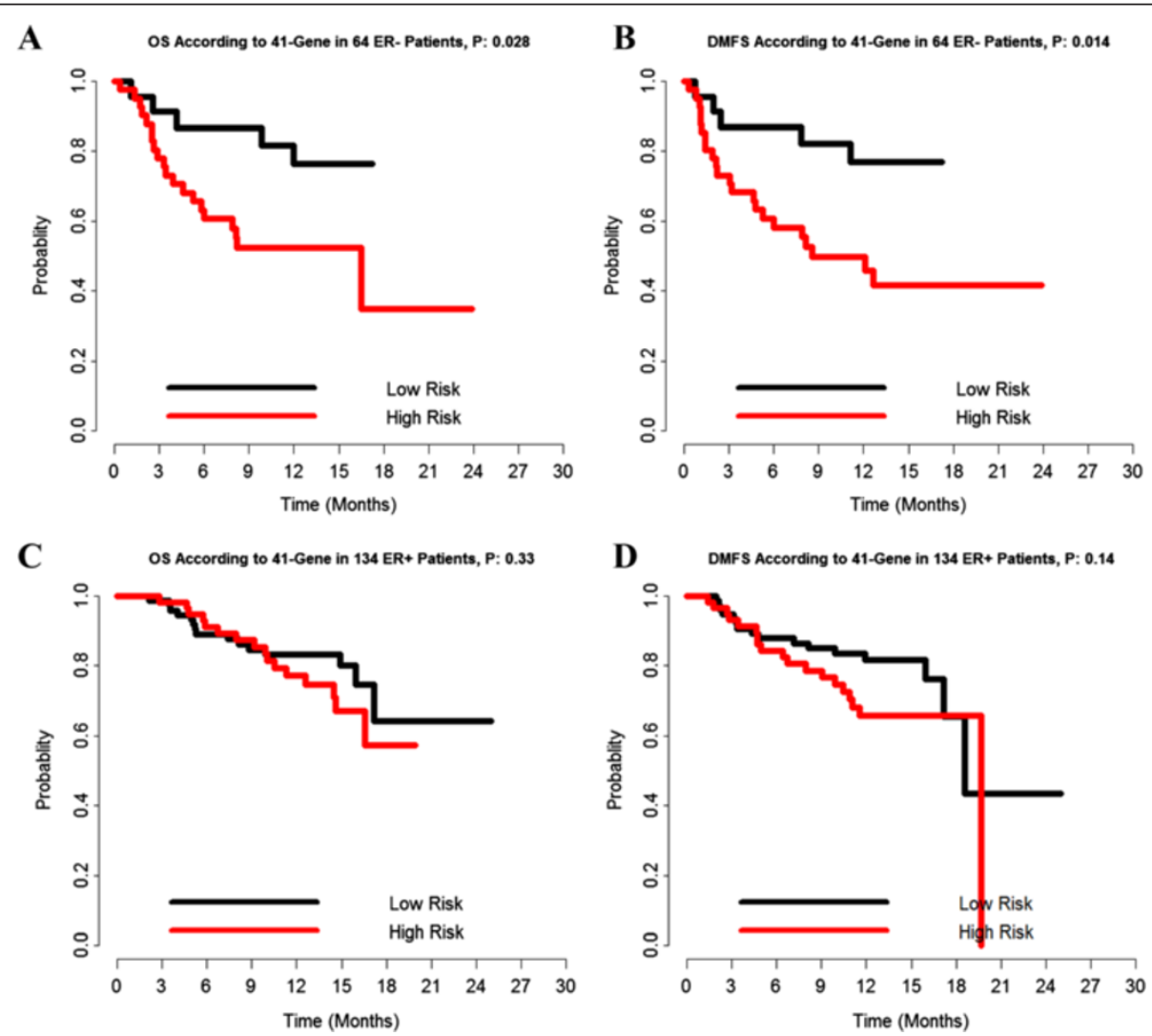

Figure 6 The prognostic value of 41-gene signature in ER positive and ER negative patients. A. prognostic value of OS in $E R^{-}$patients; B. prognostic value of DMFS in ER $R^{-}$patients; $\mathbf{C}$. prognostic value of OS in $E R^{+}$patients; $\mathbf{D}$. prognostic value of DMFS in $E R^{+}$patients. 
cancer tissues. These subgroups differ with respect to disease outcome in patients with locally advanced breast cancer. Generally, it is agreed that patients with poor prognostic features benefit most from adjuvant therapy.

We previously identified a gene expression profile of 41-gene markers that is associated with BCSCs. Since BCSCs are considered to be the root of metastasis, promote recurrence of the malignancy, and are resistant to traditional therapy [24-27], we tested this profile in a series of 198 consecutive patients who were diagnosed with early breast cancer. The results showed that the 41gene profile performed best as a predictor of DMFS by classifying patients into high-EL and low-EL groups. The prognostic signature is also a strong predictor of OS in patients with lymph node negative disease in this cohort.

To our knowledge, this is the first attempt at using cancer stem cell related markers as a prognostic signature predicting the survival and recurrence of breast cancer patients. This finding is important since the presence of cancer stem cells is a strong predictor of poor survival and resistance to traditional therapy. This finding also sheds new light on the common biological processes relevant for predicting outcome in breast cancer.

Comparing Figure 2A and Figure 3A, we see a strong correlation between the good-prognostic signature and DMFS $(P=.002)$. Similar results were observed in the analysis of OS $(P=.0086)$. To obtain a more useful estimate of clinical outcome, we calculated the probability of patients who remained free of distant metastasis and OS according to the prognosis profile. For this analysis, our results indicated that the prognostic signature was highly predictive of the risk of distant metastasis. Prolonged OS was also observed in patients with low expression of the 41-gene signature compared to patients in the high-EL group. These results highlight the value of prognostic profiles and the robustness of the profiling technique.

For the purpose of comparison, we also analyzed wellestablished criteria currently used in the clinic predicting clinical outcomes for breast cancer patients, such as AOL, NPI, St. Gallen, and Veridex. Figure 2 and Figure 3 shows the Kaplan-Meier estimates of the probability that patients would remain free of distant metastasis and OS among the 198 patients with lymph-node-negative breast cancer. In these analysis, patients were classified either by the 41-gene-expression profile or by another commonly used criteria, such as AOL, NPI consensus criteria, St. Gallen criteria, or Veridex criteria. The results indicated that only the NPI consensus criteria $(P=.0172)$ predicted a statistically significant survival outcome in this cohort. It is worth noting that no statistical significance was observed for AOL, NPI, or St. Gallen criteria in predicting clinical outcome for this cohort of breast cancer patients.
MammaPrint [28] and Oncotype Dx [29] are currently commercially available diagnostic tests that quantify the likelihood of disease recurrence in women with earlystage breast cancer. Within this cohort, the analysis revealed that the 41-gene signature and Oncotype Dx both had strong prognostic value in predicting DMFS and OS in this 198 patient group. However, there was no statistically significant difference observed for the analysis with MammaPrint.

High-risk patients identified by AOL, NPI, St. Gallen, or Veridex criteria tended to have a lower likelihood of DMFS and OS than those classified according to the 41gene expression profiling. This result indicates that both sets of the currently used criteria "misclassified" a clinically significant number of patients. Indeed, the high-risk group, defined according to these criteria, might include a number of patients who actually had a good-prognostic signature with a possible good outcome. Since both these subgroups contain some "misclassified" patients (who can be better identified through the prognosis signature), these patients might be mistreated in current clinical practice.

Based on our analysis, we predict that the 41-gene signature profile significantly associates with clinical outcome in the entire patient cohort. Thus, we further evaluated the prognostic utility of these 41-genes in ER positive and ER negative patients, respectively. In the subgroup analysis, there was a significant association between the 41-gene signature and both OS and DMFS in ER-negative breast cancer patients. In contrast, the signature did not show strong predictive ability for ER positive patients.

The molecular mechanisms regulating BCSCs are distinct from the mechanisms governing differentiated tumor cells. Our data indicate that classification of patients into high-risk and low-risk subgroups on the basis of the 41-gene prognostic profile could prove to be a very useful means of guiding adjuvant therapy in patients with lymph-node-negative breast cancer. This approach should also improve the selection of patients who would benefit from adjuvant systemic treatment, reducing the rate of both over-treatment and under-treatment. Even though these results are encouraging, a larger scale prospective study is required to confirm these results.

\section{Conclusion}

The 41-gene prognostic profile demonstrates prognostic significance with strong capability of predicting DMFS and $\mathrm{OS}$ in node-negative breast cancer patients. This 41-gene signature of BCSCs was even more strongly associated with clinical outcomes compared with other existing criteria, such as AOL, NPI, Veridex, St. Gallen, and MammaPrint.

Competing interests

There are no competing interests among the authors. 


\section{Authors' contributions}

YZQ, LJ , XYC, FY, TL, XYW, LXL, MY collected clinical information. XYW, YJ, DGH and LBH performed the statistical analysis. YZQ, LJ and WHX participated in the design of the study. XYC and YJ drafted the manuscript. WHX revised the manuscript. All authors read and approved the final manuscript.

\section{Acknowledgements}

This study was supported by the National Natural Science Funds (Project Number: 81102015), the National Program on Key Basic Research Project (973 Program) (Project Number: 2013CB967201), Shanghai Health Bureau Key Disciplines and Specialties Foundation and the Special Funds for Technological Innovation of Shanghai Jiaotong University (Project Number: YG2012MS46)

\section{Author details}

'Shanghai Renji Hospital, Shanghai Jiaotong University School of Medicine, Shanghai 200127, China. ${ }^{2}$ Shanghai Center for Bioinformation Technology, Shanghai 201203, China. ${ }^{3}$ Department of Oncology, Renji Hospital, School of Medicine, Shanghai Jiaotong University, Shanghai 200127, China.

Received: 7 January 2014 Accepted: 15 April 2014

Published: 6 June 2014

\section{References}

1. Wang Y, Klijn JG, Zhang Y, Sieuwerts AM, Look MP, Yang F, Talantov D, Timmermans M, Meijer-van Gelder ME, Yu J, Jatkoe T, Berns EM, Atkins D, Foekens JA: Gene-expression profiles to predict distant metastasis of lymph-node-negative primary breast cancer. Lancet 2005, 365:671-679.

2. Bast RC Jr, Ravdin P, Hayes DF, Bates S, Fritsche H Jr, Jessup JM, Kemeny N, Locker GY, Mennel RG, Somerfield MR: 2000 update of recommendations for the use of tumor markers in breast and colorectal cancer: Clinical practice guidelines of the American Society of Clinical Oncology. J Clin Oncol 2001, 19:1865-1878.

3. Peng G, Zhihua Y, Xuelian L, Wei W, Baosen Z: Meta-analysis of human lung cancer microRNA expression profiling studies comparing cancer tissues with normal tissues. JECCR 2012, 31:54.

4. Wacholder S, Hartge P, Prentice R, Garcia-Closas M, Feigelson HS, Diver WR Thun MJ, Cox DG, Hankinson SE, Kraft P, Rosner B, Berg CD, Brinton LA, Lissowska J, Sherman ME, Chlebowski R, Kooperberg C, Jackson RD, Buckman DW, Hui P, Pfeiffer R, Jacobs KB, Thomas GD, Hoover RN, Gail MH, Chanock SJ, Hunter DJ: Performance of common genetic variants in breast-cancer risk models. N Engl J Med 2010, 362:886-893.

5. Anders CK, Deal AM, Miller CR, Khorram C, Meng H, Burrows E, Livasy C, Fritchie K, Ewend MG, Perou CM, Carey LA: The prognostic contribution of clinical breast cancer subtype, age, and race among patients with breast cancer brain metastases. Cancer 2011, 8:1602-1611.

6. Comen EA, Norton L: Breast cancer tumor size, nodal status, and prognosis: biology trumps anatomy. J Clin Oncol 2011, 23:2610-2612

7. Ma XJ, Wang Z, Ryan PD, Isakoff SJ, Barmettler A, Fuller A, Muir B, Mohapatra G, Salunga R, Tuggle JT, Tran Y, Tran D, Tassin A, Amon P, Wang W, Wang W, Enright E, Stecker K, Estepa-Sabal E, Smith B, Younger J, Balis U, Michaelson J, Bhan A, Habin K, Baer TM, Brugge J, Haber DA, Erlander MG, Sgroi DC: A two-gene expression ratio predicts clinical outcome in breast cancer patients treated with tamoxifen. Cancer Cell 2004, 5:607-616.

8. Cronin-Fenton DP, Christensen M, Lash TL, Ahern TP, Pedersen L, Garne JP, Ewertz M, Autrup H, Sørensen HT, Hamilton-Dutoit S: Manganese superoxide dismutase and breast cancer recurrence: a Danish clinical registry-based case-control study, and a meta-analysis. PLoS One 2014, 9:87450

9. O'Brien CA, Pollett A, Gallinger S, Dick JE: A human colon cancer cell capable of initiating tumour growth in immunodeficient mice. Nature 2006, 445:106-110

10. Ricci-Vitiani L, Lombardi DG, Pilozzi E, Biffoni M, Todaro M, Peschle C, De Maria R: Identification and expansion of human colon-cancer-initiating cells. Nature 2006, 445:111-115.

11. Dalerba P, Cho RW, Clarke MF: Cancer stem cells: models and concepts. Annual Rev Med 2007, 58:267-284.

12. Manale El I, Valérie H, Philippe M, Franck M, Eric W: Cause-specific telomere factors deregulation in hepatocellular carcinoma. JECCR 2013, 32:64.
13. Wang H, Huang M, Zhang DY, Zhang F: Global profiling of signaling networks: study of breast cancer stem cells and potential regulation. Oncologist 2011, 16:966-979.

14. Zhang F, Song C, Ma Y, Tang L, X Y Y, Wang H: Effect of fibroblasts on breast cancer cell mammosphere formation and regulation of stem cell-related gene expression. Int J Mol Med 2011, 28:365-371.

15. Bland JM, Altman DG: The logrank test. BMJ 2004, 328:1073.

16. Desmedt C, Piette F, Loi S, Wang Y, Lallemand F, Haibe-Kains B, Viale G, Delorenzi M, Zhang Y, d'Assignies MS, Bergh J, Lidereau R, Ellis P, Harris AL, Klijn JG, Foekens JA, Cardoso F, Piccart MJ, Buyse M, Sotiriou C: Strong time dependence of the 76-gene prognostic signature for node-negative breast cancer patients in the TRANSBIG multicenter independent validation series. Clin Cancer Res 2007, 13:3207-3214.

17. van de Vijver MJ, He YD, van't Veer LJ, Dai H, Hart AA, Voskuil DW, Schreiber GJ, Peterse JL, Roberts C, Marton MJ, Parrish M, Atsma D, Witteveen A, Glas A, Delahaye L, van der Velde T, Bartelink H, Rodenhuis S, Rutgers ET, Friend $\mathrm{SH}$, Bernards R: A gene-expression signature as a predictor of survival in breast cancer. N Engl J Med 2002, 347:1999-2009.

18. Sotiriou C, Phil D: Gene-expression signatures in breast cancer. N Engl J Med 2009, 360:790-800.

19. Devilee P, Rookus MA: A tiny step closer to personalized risk prediction for breast cancer. N Engl J Med 2010, 362:1043-1045.

20. Yunzhen $C$, Haichun L, Wenliang W, Yi L, Jianmin L: Osteopontin genetic variants are associated with overall survival in advanced non-small-cell lung cancer patients and bone metastasis. JECCR 2013, 32:45.

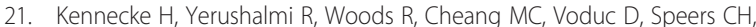
Nielsen TO, Gelmon K: Metastatic behavior of breast cancer subtypes. J Clin Oncol 2010, 28:3271-3277.

22. Paik S, Tang G, Shak S, Kim C, Baker J, Kim W, Cronin M, Baehner FL, Watson D, Bryant J, Costantino JP, Geyer CE Jr, Wickerham DL, Wolmark N: Gene expression and benefit of chemotherapy in women with node-negative, estrogen receptor-positive breast cancer. J Clin Oncol 2006, 24:3726-3734.

23. Perou CM, Sørlie T, Eisen MB, van de Rijn M, Jeffrey SS, Rees CA, Pollack JR, Ross DT, Johnsen H, Akslen LA, Fluge O, Pergamenschikov A, Williams C, Zhu SX, Lønning PE, Børresen-Dale AL, Brown PO, Botstein D: Molecular portraits of human breast tumors. Nature 2000, 406:747-752.

24. O'Neill JD: Concise review: transmissible animal tumors as models of the cancer stem-cell process. Stem Cells 2011, 29:1909-1914.

25. Olsson E, Honeth G, Bendahl PO, Saal LH, Gruvberger-Saal S, Ringnér M, Vallon-Christersson J, Jönsson G, Holm K, Lövgren K, Fernö M, Grabau D, Borg A, Hegardt C: CD44 isoforms are heterogeneously expressed in breast cancer and correlate with tumor subtypes and cancer stem cell markers. BMC Cancer 2011, 11:418.

26. Borovski T, De Sousa E, Melo F, Vermeulen L, Medema JP: Cancer stem cell niche: the place to be. Cancer Res 2011, 71:634-639.

27. Jordan CT, Guzman ML, Noble M: Cancer stem cells. N Engl J Med 2006, 355:1253-1261.

28. Veer LJ V 't, Dai H, van de Vijver MJ, He YD, Hart AA, Mao M, Peterse HL, van der Kooy K, Marton MJ, Witteveen AT, Schreiber GJ, Kerkhoven RM, Roberts C, Linsley PS, Bernards R, Friend SH: Gene expression profiling predicts clinical outcome of breast cancer. Nature 2002, 415:530-536.

29. Paik S, Shak S, Tang G, Kim C, Baker J, Cronin M, Baehner FL, Walker MG, Watson D, Park T, Hiller W, Fisher ER, Wickerham DL, Bryant J, Wolmark N: A multigene assay to predict recurrence of tamoxifen-treated, node-negative breast cancer. N Engl J Med 2004, 351:2817-2826.

doi:10.1186/1756-9966-33-49

Cite this article as: Yin et al:: A 41-gene signature derived from breast cancer stem cells as a predictor of survival. Journal of Experimental \& Clinical Cancer Research 2014 33:49. 